\title{
Rôle et place du non-verbal dans la rationalisation des projets multimédias
}

Julien Huart, Alain Durand et Sylvie Leleu-Merviel

\section{OpenEdition}

Journals

Édition électronique

URL : https://journals.openedition.org/communicationorganisation/2470

DOI : 10.4000/communicationorganisation.2470

ISSN : $1775-3546$

\section{Éditeur}

Presses universitaires de Bordeaux

\section{Édition imprimée}

Date de publication : 1 novembre 2000

ISSN : 1168-5549

Référence électronique

Julien Huart, Alain Durand et Sylvie Leleu-Merviel, « Rôle et place du non-verbal dans la rationalisation des projets multimédias », Communication et organisation [En ligne], 18 | 2000, mis en ligne le 27 mars 2012, consulté le 05 août 2021. URL : http://journals.openedition.org/communicationorganisation/ 2470 ; DOI : https://doi.org/10.4000/communicationorganisation.2470

Ce document a été généré automatiquement le 5 août 2021.

(C) Presses universitaires de Bordeaux 


\title{
Rôle et place du non-verbal dans la rationalisation des projets multimédias
}

\author{
Julien Huart, Alain Durand et Sylvie Leleu-Merviel
}

\section{Introduction}

1 Communiquer fait intervenir deux types de signaux : verbaux et non verbaux. Dans le cas d'une communication médiatisée (comme par exemple par le biais du document multimédia) ces deux dimensions se confrontent. Alors que jusqu'à présent les différents médias se marquaient par une prédominance d'une dimension sur l'autre, le multimédia les associe pratiquement à part égale. Cela ne va pas sans créer des problèmes de création et de rationalisation de l'organisation dans les projets de conception et de réalisation multimédias. Deux termes semblent s'opposer, rigueur et créativité, rationalité et subjectivité. En ce sens, deux types de processus créatifs, l'un d'apparence désorganisée et de nature plutôt non-verbale, l'autre rigoureux et à prédominance verbale peuvent être considérés.

2 D'un côté se trouvent les processus qui correspondent à l'évaluation/modification d'un seul prototype tout au long de la création : c'est le cas notamment de la peinture. Dans ce cas, la réflexion et l'action humaines sont directement génératrices d'un résultat observable, et donc évaluable, qui permet à la créativité de s'exprimer en retour, par feedback. S'y opposent les processus qui, à l'inverse, nécessitent une planification, ce qui contraint notamment à passer par des phases de conception avant de produire : le document audiovisuel, le programme informatique en sont des exemples. On se trouve ici dans le cas d'une création dont on ne voit pas le résultat au fur et à mesure de l'avancée du projet.

3 Les projets multimédias actuels tendent à répondre au premier processus évoqué du fait de lacunes méthodologiques et de méconnaissance des propriétés du multimédia par les professionnels du secteur; le non-verbal y joue un rôle prépondérant. 
Cependant, les documents réalisés sont généralement de qualité médiocre et la créativité ne semble pas pouvoir réellement s'exprimer. Cette communication a pour but de montrer que créativité, contraintes et rigueur doivent aller de pair pour la fabrication de documents multimédias, associant à cet effet verbal et non-verbal. Les projets multimédias gagnent donc à incliner plutôt vers le second processus présenté.

Dans un premier temps, le document multimédia et ses caractéristiques majeures seront présentés, mettant en relief la multidisciplinarité nécessaire à la conception et la réalisation de tels documents, et le rôle du verbal et du non-verbal dans les organisations actuelles. Les problèmes méthodologiques majeurs seront ensuite évoqués, insistant sur le besoin de rationalisation du processus, de partage respectif des tâches entre les acteurs du projet, et d'organisation de leur interaction.

Dans ce but, la notion de projet, dont le cadre est défini par un objectif relié à la satisfaction des attentes et/ou besoins relatifs à un document, sera mise en avant. Les outils usuels de la qualité (analyse fonctionnelle. Cahier des Charges Fonctionnel...) s'avèrent appropriés pour ce faire. La maîtrise du cycle de vie des documents multimédias sera finalement envisagée.

\section{Définition du document multimédia}

6 Le document multimédia constitue une nouvelle forme de transmission de l'information qui s'appuie à la fois sur le verbal et le non-verbal. Il n'en conserve pas moins les caractéristiques majeures du document.

\section{Définition de la notion de document}

7 La préparation d'un contenu informationnel finalisé consiste à sélectionner les données retenues et à les organiser, c'est-à-dire à définir une structure élaborée comportant un ensemble de sous parties, elles-mêmes décomposables jusqu'au niveau de la donnée élémentaire. L'appellation "document» désigne précisément cette structure organisationnelle.

8 Définition : un document est une structure organisée de fragments informationnels de niveau moindre ${ }^{1}$.

9 Le document est notamment utilisé lorsqu'il y a rupture temporelle dans la communication. La raison est qu'il se marque par une double indépendance par rapport au temps : la synchronie et la stabilité 2 . Le document a donc été utilisé, à travers les siècles, par inscription sur un support mobile et léger qui permettait son transport. Ce support lui donnait également la possibilité d'être reproduit, même si l'on peut noter que le document est indépendant de celui-ci : «le terme document désigne ainsi la structure qui régit l'organisation de sous parties constituantes, et ceci en totale indépendance vis-à-vis de l'objet physique réalisé en final $»^{3}$.

\section{Composantes verbales et non-verbales de la communication}

10 Les signaux analogiques ont été les premiers signes utilisés par l'homme: ils comprennent les actions ainsi que le paralangage : ces signes ne renvoient pas à des codes précis, même si l'expérience et le contexte interviennent dans leur 
compréhension. La communication analogique n'est pas guidée par la conscience et la volonté; elle relève plus de l'inconscient et de l'intuitivité. On lui attribue donc également le nom de communication relationnelle ou de non-verbale, puisqu'elle échappe à la maîtrise du niveau conscient. D'après Alex Mucchielli, elle fonctionne «sur le mode de l'expérience totale, intuitive et indicible $»^{4}$.

11 Les signaux digitaux sont apparus plus tard dans l'histoire humaine, et correspondent aux mots: le langage en est le support. La communication digitale ou verbale correspond au contenu proprement dit des échanges: elle relève de la conscience et donc aussi de l'analyse. Toute communication interpersonnelle consiste en un mélange du verbal et du non-verbal. Dans la communication médiatisée, on retrouve également ces différents équilibres entre le verbal et le non-verbal : c'est le cas notamment du multimédia.

\section{Notion de multimédia et d'hyperdocument}

Définition : le multimédia est une technologie de communication qui tend à rassembler sur des supports de même type l'ensemble des données plurisensorielles (niveau physique) et informatiques (niveau logique) ${ }^{5}$.

13 Le document multimédia est considéré comme un contenu informationnel pouvant être supporté par un medium. Il se marque, de plus, par une multiplicité de parcours et la possibilité pour l'utilisateur d'intervenir directement sur la diffusion des contenus, ce que définit la notion d'hyperdocument :

14 Définition : un hyperdocument est un contenu informatif constitué d'une nébuleuse de fragments dont le sens se construit à travers chacun des parcours que la lecture détermine ${ }^{6}$.

15 Certains médias constituent des supports privilégiés pour le contenu et donc l'expression rationnelle. C'est évidemment le cas des « descendants» de la machine à imprimer inventée par Gutenberg : le livre, la presse, etc.

D'autres médias beaucoup plus récents sont complètement tournés vers l'image : les bandes dessinées, la télévision, le cinéma... Notons que la peinture, la musique, sont aussi à cette extrémité de l'intervalle entre une communication verbale et une communication non-verbale.

17 Considérant le document multimédia et son mode de lecture actuel (l'utilisateur est généralement devant un poste informatique), il apparait plusieurs points: le multimédia utilise beaucoup la perception humaine; bref, il agit assez fortement sur l'inconscient et sa composante relationnelle ne fait aucun doute. Néanmoins, la présence de texte, d'actions à effectuer et la position et l'environnement de lecture favorisent plutôt un comportement rationnel et actif qu'un état hypnoïde. Par conséquent, sa place est proche du centre d'un intervalle entre une communication digitale totale et une communication analogique totale (cf. figure 1). 


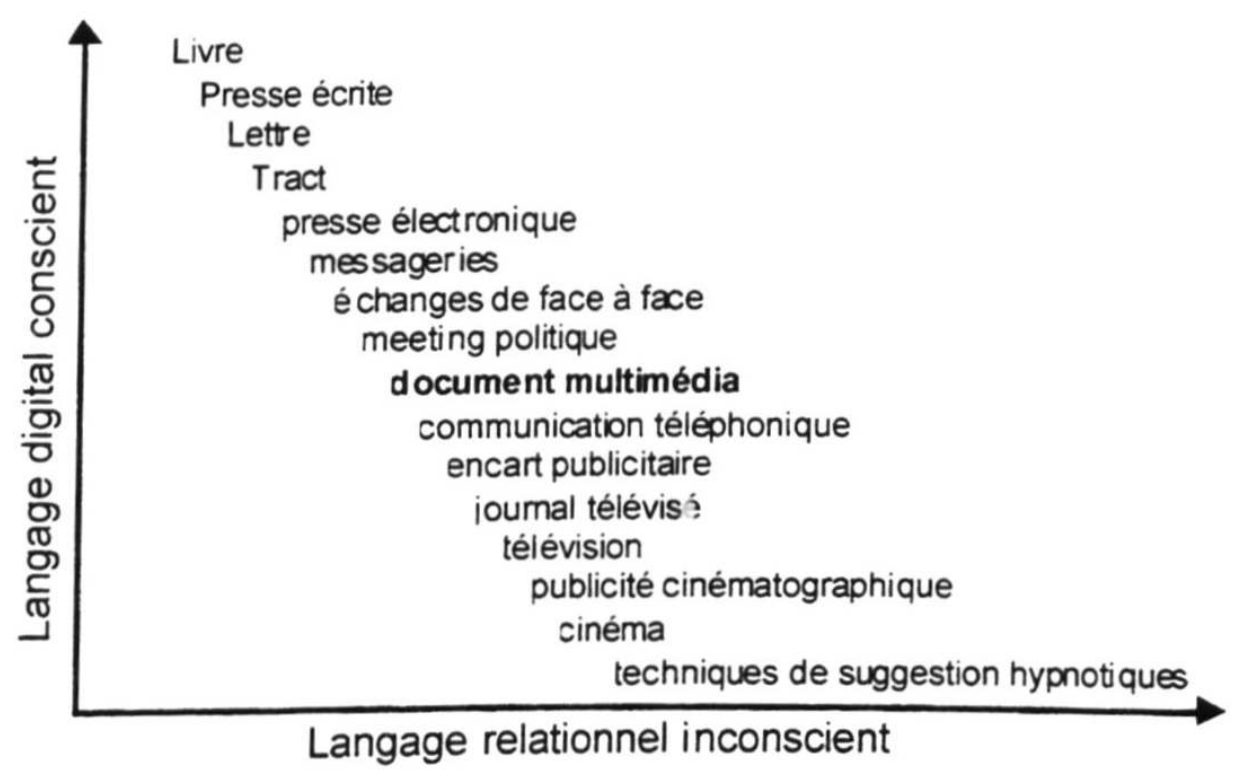

Figure 1 : Les médias et le multimédia par rapport aux deux dimensions de la communication (d'après Alex Mucchielli). création de documents multimédias : les projets multimédias rassemblent
venant de disciplines relativement variées, préoccupés par des aspects
document à créer, mais surtout s'appuyant sur des méthodes de travall
non-verbales) différentes sinon opposées. Cela ne va pas sans créer un cert
de problèmes méthodologiques.
Problèmes méthodologiques liés à la création
d'hyperdocuments professionnels actuels à suivre une démarche plutôt non-verbale, plus ou moins imposée par « l'élan » de créativité, entraîne de sérieuses conséquences sur les projets.

\section{Processus humain de création}

Lorsqu'il cherche à résoudre un problème ou à créer, l'homme effectue une action puis en évalue le résultat jusqu'à atteindre un résultat qui le satisfait ${ }^{7}$. Tout se passe finalement de façon assez proche du processus suivant :

Dans une première étape, l'homme conçoit à partir d'idées, de projections qu'il fait du futur produit de son imagination. 11 effectue finalement une action.

Dans un second temps, il perçoit une modification de son environnement et cherche à déterminer si cette évolution a été globalement dans le sens qu'il souhaite. Le résultat perçu est comparé au résultat précédemment envisagé. Selon les résultats de cette comparaison s'engage alors un nouveau cycle action-reconnaissance.

Ce principe de création est tout à fait non-verbal et correspond par exemple en tout point aux activités d'un artiste créant, seul, une œuvre.

Communication et organisation, 18 | 2000 


\section{Application au multimédia}

L'individu pensant émet tout d'abord un concept. Le concept a notamment pour caractéristiques d'être une abstraction à partir des sens, une production de l'esprit, et d'être réutilisable.

Définition: un concept est une représentation intellectuelle d'un objet conçu par l'esprit. ${ }^{8}$

Le concept est, comme on l'a vu plus haut, instancié pour donner naissance à une partie du message, du produit. L'instance ainsi créée est évaluée: les résultats de cette évaluation, sensations ou réflexions conduisent l'individu à reconceptualiser tout ou partie du message.

7 Une autre stratégie, plus élaborée, peut être mise en œuvre pour la création, même si elle s'apparente plus à une stratégie de résolution de problèmes : la planification ${ }^{9}$. La planification permet de faire l'économie du tâtonnement caractéristique du processus de conception/instanciation et aboutit à une action rationnelle, mais qui n'est plus forcément artistique. Elle correspond généralement à des domaines matériellement et techniquement plus complexes.

On peut donc distinguer, à partir de leur méthodologie de conception et de réalisation, deux types de produits :

Les produits qui sont réalisés par l'évaluation/modification d'un seul prototype tout au long de la création. Dans ce cas. la réflexion et l'action humaines sont directement génératrices d'un résultat observable et donc évaluable.

Les produits dont la fabrication nécessite une planification, ce qui contraint notamment à passer par des phases de conception avant de produire. On se trouve ici dans le cas d'une création dont on ne voit pas simultanément le résultat.

1 Les produits multimédias correspondent à ce second type ; cependant, comme pour toute discipline jeune, il n'existe ni expérience, ni formalismes rigoureux et systématiques. Les auteurs ont donc tendance à faire converger leur méthodologie vers le premier cas présenté ci-dessus et à favoriser des échanges non-verbaux.

\section{Conséquences sur la création multimédia}

Les projets multimédias présentent donc très souvent les caractéristiques suivantes :

- une étude des risques limitée,

- une absence de planification des activités de production,

- une limitation des évaluations de produits fournis, des erreurs de production.

- une absence de documentation et de traçabilité des projets,

- une absence d'analyse et de conception,

- un oubli de la cible dans toutes les étapes du cycle de vie,

- enfin, un non-respect de l'ordre normal des étapes de conception et réalisation.

La création se trouve finalement bridée par l'ensemble de ces problèmes et les conséquences sur les documents sont bien souvent désastreuses ${ }^{10}$ et menacent même souvent la pérennité des organisations qui supportent leur fabrication. Pour résorber 
ce déficit méthodologique, de nombreux auteurs ont montré qu'il faut rationaliser les processus de conception et de réalisation ${ }^{11}{ }^{12}$.

\section{Rationalisation des projets multimédias}

41 Corriger les défauts actuels signifie organiser et planifier les activités, favoriser les échanges d'informations entre tous les intervenants des projets et assurer le suivi du produit tout au long de son cycle de vie. Pour ce faire, les outils de la qualité offrent un support idéal.

\section{Notion de programme : les méthodes de fabrication}

Comme nous l'avons vu précédemment, dès que les projets sont lourds et complexes, il convient de les planifier et d'organiser le cheminement à travers des étapes de réalisation. Tout processus complexe doit être décomposé en sous-processus plus simples, et ceci par raffinements successifs jusqu'à obtention de processus élémentaires aisément maîtrisables : le programme correspond à cette décomposition.

Définition: un programme est un ensemble organisé (éventuellement formalisé) d'actions et/ou d'opérations nécessaires et suffisantes pour atteindre un résultat, à savoir la réalisation d'un produit.

Le programme est découpé en quelques phases majeures qui sont représentées sur la figure 2 :

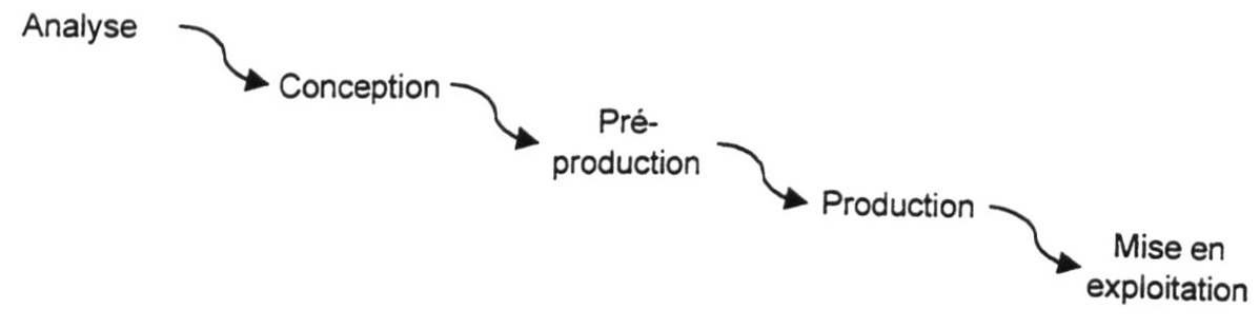

Figure 2 : Principales étapes d'un programme

Ces étapes classiques se retrouvent dans tous les projets de grande échelle. Leur organisation se complexifie néanmoins avec ces derniers.

\section{Conséquences sur la création}

Il est naturel de penser que des logiques de fabrication plus élaborées puissent limiter la créativité. Cependant, ces logiques de fabrication permettent toujours à l'esprit humain d'agir sur ses concepts en suivant le processus de conception/instanciation : la créativité, quoique guidée (elle ne s'exprime pas de façon diffuse et désordonnée), reste intacte. Par exemple, l'OuLiPo (Ouvroir de la Littérature Potentielle) et notamment Georges Perec avec « La disparition » ont montré que l'existence d'un corpus de règles était un fondement créatif de l'écriture.

De plus, comme l'ont montré les paragraphes précédents, la créativité n'est pas un simple «laisser penser». Elle s'apparente davantage à l'organisation d'un «mieux 
penser ». Il est assez fréquent que la délimitation d'un cadre très strict fasse émerger des degrés de liberté inédits. L'histoire montre d'ailleurs que des périodes de forte créativité sont nées de l'inconfort, la pénurie, la guerre ou encore des privations de tous ordres.

Dans ce sens, la collaboration entre créatifs et non créatifs (notamment gestionnaires) peut être fructueuse lorsqu'il y a répartition des tâches. L'inventeur et le critiqueur apportent tous deux au projet et au produit des ressources asymétriques. L'existence de règles de collaboration garantissant une certaine autonomie des acteurs du projet permettent de réguler le processus. Ces règles, de nature verbale, sont fournies notamment par les outils de la qualité.

\section{Les outils de la qualité}

Définition : la qualité désigne l'ensemble des propriétés et caractéristiques d'un produit ou service qui lui confèrent l'aptitude à satisfaire des besoins exprimés ou implicites ${ }^{13}$.

50 L'un des principes forts de l'accession à la qualité repose sur la séparation du besoin à satisfaire et des solutions retenues pour répondre à ce besoin ${ }^{14}$. Des directives précises permettent d'élaborer l'expression fonctionnelle du besoin, qui conduit à l'élaboration d'un Cahier des Charges Fonctionnel. Celles-ci constituent le premier stade de la démarche qualité, répondant à l'objectif prioritaire :

51 - identifier les besoins.

52 Une fois réalisée l'analyse fonctionnelle, le processus entre dans sa seconde phase, à savoir la recherche d'une solution adaptée. On se trouve alors au niveau de l'ingénierie. Il s'agit de satisfaire le second objectif :

53 - améliorer les solutions qui découlent.

54 Puis, le processus suivra successivement les stades de production et d'exploitation. Pendant le déroulement des étapes ultérieures à l'analyse fonctionnelle, il importe de continuer à contrôler la qualité en permanence. Cette exigence se traduit par un troisième objectif à atteindre :

55 - identifier les risques de dysfonctionnement et les réduire.

56 Il en résulte que la recherche de la qualité implique le respect de directives générales permettant de garantir la maîtrise de la qualité tout au long du cycle de vie du produit.

57 Un ensemble très vaste d'activités variées, liées à la réalisation de produits différents, a permis d'extraire une architecture de programme commune. On peut donc penser que cette architecture structure le processus de production lui-même, indépendamment du type de produit considéré. L'architecture de programme générique formalise les états successifs du produit au cours de son cycle de vie. Les processus de transition d'état à état constituent un premier niveau de décomposition du programme en opérations. Tous ces éléments peuvent être consignés dans un diagramme états-transitions, qui schématise l'ensemble du cycle de vie d'un produit (cf. figure 3). 


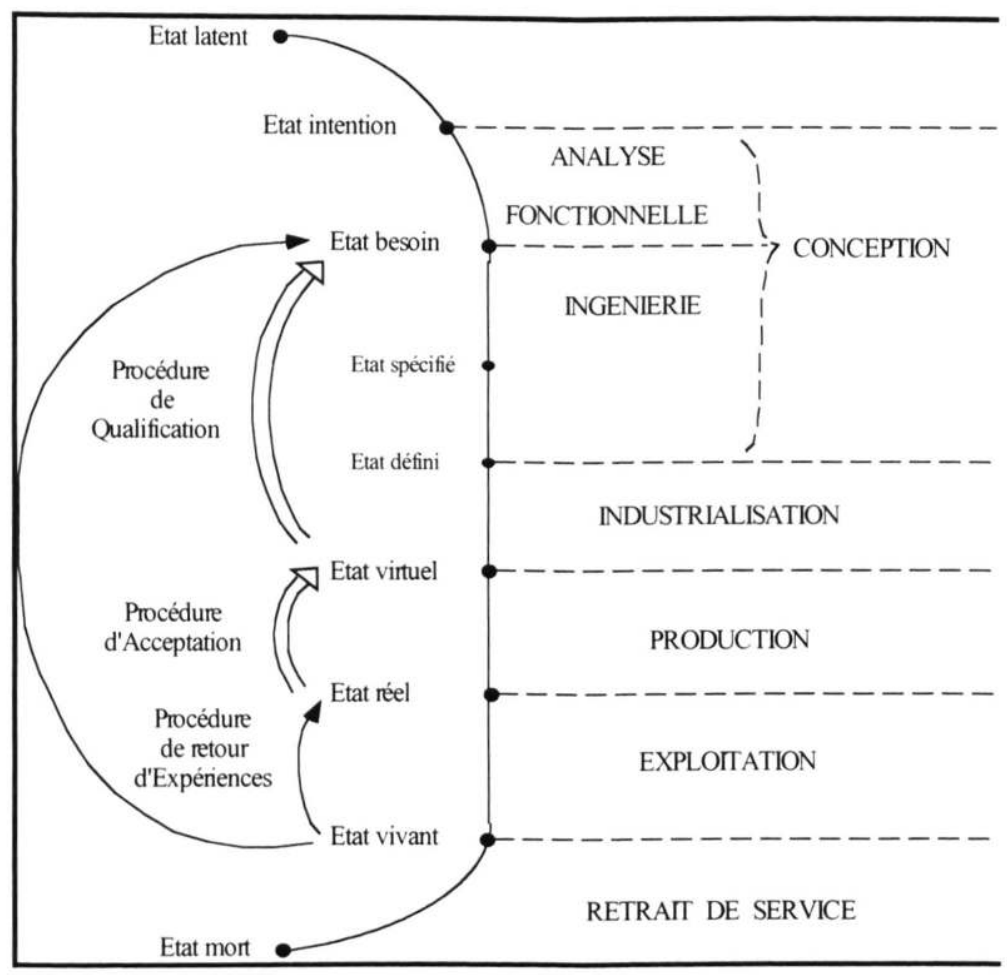

Figure 3 : Architecture générale d'un programme conforme à l'approche qualité.

La disposition séquentielle des phases les unes par rapport aux autres suggère un certain ordre temporel à respecter. Toutefois, leur enchainement dans le temps n'est jamais rigoureusement linéaire.

\section{Maîtrise du cycle de vie de documents multimédias : le programme AUTHOR}

Le programme AUTHOR pour Authoring and User oriented meThodology for Hypermedia modeling and cReation (Méthodologie orientée Création et Utilisateur pour la Conception et la Réalisation d'Hyperdocument) a pour objet d'offrir un cadre méthodologique adapté au milieu professionnel et a pour but de favoriser la créativité de l'auteur et les échanges d'information entre les différents intervenants d'un projet multimédia.

Il s'appuie sur les interconnections d'étapes majeures inspirées de la démarche qualité :

61 - L'analyse préalable: extraire du discours du commanditaire le besoin réel est essentiel et conduit au cahier des charges préliminaire. L'analyse fonctionnelle conduit au cahier des charges fonctionnel, qui consigne les résultats de l'analyse.

62 - La conception préliminaire : les grandes lignes du produit sont établies. Le modèle utilisateur et le modèle des tâches sont créés. Les choix matériels et les études financières et du risque sont également faits à ce moment.

63 - La conception détaillée fait aboutir la réflexion conceptuelle : les chartes (graphiques, de navigation,...) sont définies. Le scénario est écrit. Le produit est défini. 
64 - La préproduction structure les éléments (découpage) et prépare à la production des différents médias.

- La production est validée par un premier prototype représentatif du produit final: des retours à des activités des étapes antérieures sont envisageables.

66 - L'implémentation conduit à l'état réel : des tests importants (internes, utilisateurs, commanditaires) sont réalisés.

67 - La mise en service (distribution) peut être de différents types. Elle doit cependant être active et prévoir les retours. - Une étude finale, liée à la mort du produit permet de garder une trace de tous les états significatifs du produit, de sa naissance à sa mort. Elle garantit la traçabilité de l'expérience acquise pour ce produit particulier.

68 Néanmoins, pour favoriser la créativité mais aussi tenir compte des contraintes matérielles et financières du développement de documents multimédias, le programme AUTHOR s'appuie sur des formalisations de type verbal et non-verbal des états du produit, et sur des boucles de rétroaction qui permettent des remises en cause et des ajustements tout au long du cycle de vie. Ces rétroactions concernent majoritairement les travaux effectués pendant l'analyse détaillée, la conception détaillée et la production. Elles interviennent localement, à travers le prototypage qui correspond principalement à une composante non-verbale du projet et globalement par l'intermédiaire des notations qui représentent le support verbal de l'ensemble du projet.

69 Car, si l'objectif du programme AUTHOR est de favoriser l'organisation des activités par des démarches de nature verbale, il n'en respecte pas moins les impératifs de créativité et d'expression des besoins qui nécessitent l'utilisation du non-verbal. La planification et l'organisation du projet multimédia, les échanges d'informations entre chaque intervenant, ainsi que les notations permettant la traçabilité des projets utilisent donc en priorité le verbal: ces activités sont rigoureuses et ordonnées. Les rétroactions nécessaires à la bonne marche du projet s'appuient dans ce cas sur les notations : on se situe à un niveau global. En revanche, dès qu'il s'agit du document et de sa création, le programme AUTHOR offre aux auteurs toute latitude de création : les démarches nonverbales sont dans ce cas encouragées et ont pour support les divers prototypes et maquettes réalisés au cours de chaque étape, c'est-à-dire à un niveau local du projet (cf. figure 4). 


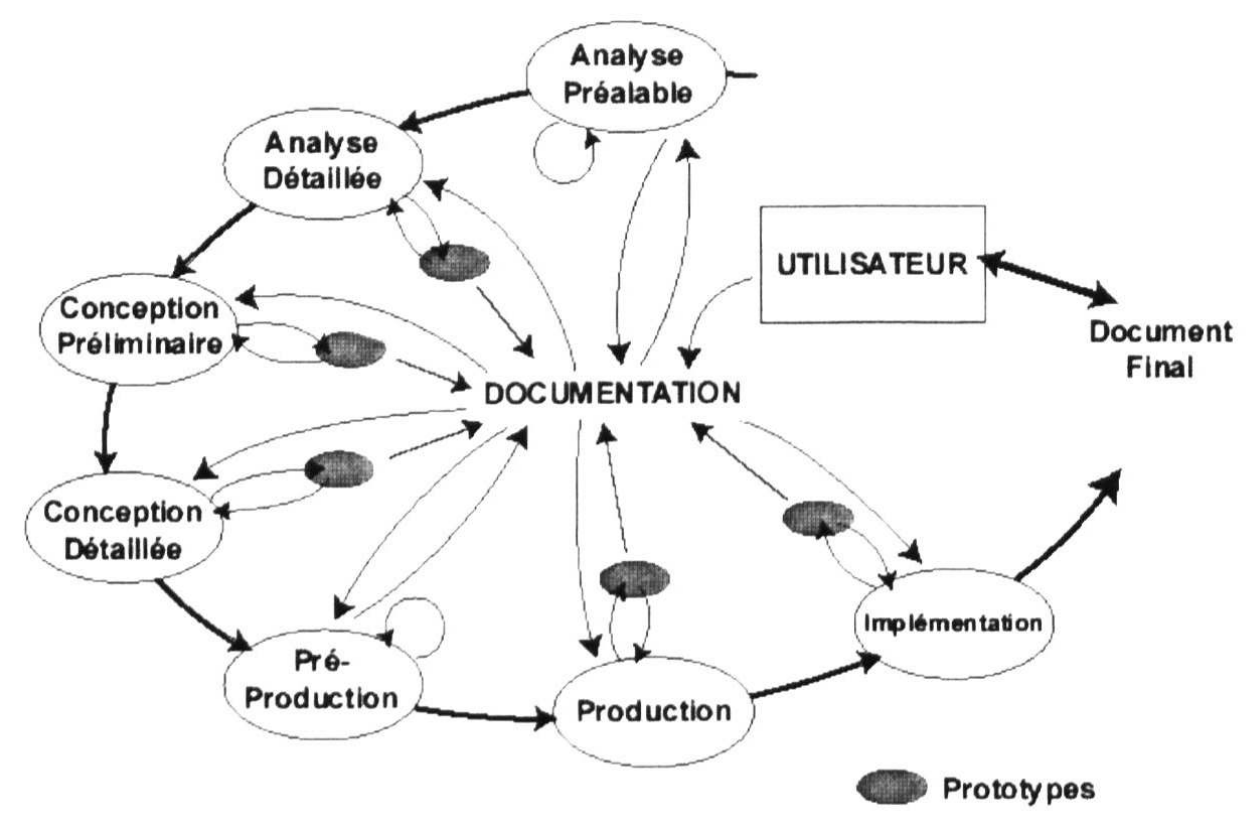

Figure 4 : Le programme AUTHOR.

\section{Conclusion} disciplines informatiques, le multimédia connaît actuellement une crise méthodologique importante. Les raisons majeures en sont une absence de documents de formalisation et une absence de culture commune du multimédia chez les différents professionnels. Cela se traduit par une absence de méthodes et par des problèmes de communication au sein de l'organisation dans laquelle le non-verbal tend à s'imposer. Le cycle de développement des documents consiste en une sorte de prototypage évolutif : les acteurs du projet, dépourvus de méthodes, se focalisent sur les outils et non sur le service à apporter.

Un certain nombre d'erreurs accompagnent donc tout projet de développement. Ces erreurs concernent principalement la gestion et la rigueur du projet, la réponse au besoin de l'utilisateur final et la prise en compte de ce dernier dans le développement. Ces points de rupture entraînent des désagréments sur le projet et le produit en construction. l'émetteur et du récepteur du message par le choix de fonctions adéquates. La La méthodologie AUTHOR propose des réponses à ces problèmes méthodologiques en
repensant la communication en cours d'élaboration des projets en direction d'un usage
équilibré et co-productif du verbal et du non-verbal. Le cycle de vie du produit conception permet d'envisager puis de définir une solution optimale. C'est à ce niveau que la composante créative s'exprime massivement, et avec elle la mise en œuvre de processus fortement ancrés dans le non-verbal. Ces étapes en amont du projet ont une grande importance pour l'ensemble de celui-ci. La pré-production, étape de transition prépare et planifie la réalisation proprement dite qui est séparée en une phase de production des données plurisensorielles et logiques et une phase d'implémentation production des données plurisensorielles et logiques et une phase d'implementation commence par des étapes d'analyse qui garantissent une bonne réponse aux besoins de

Déption

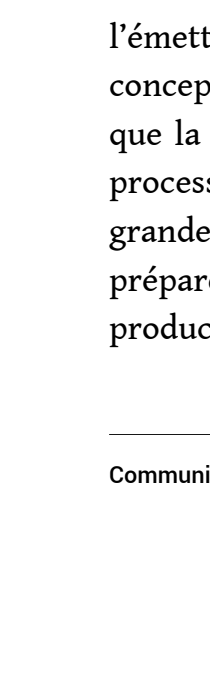


qui conduit au produit fini. Cette suite d'activités présente l'avantage d'optimiser l'organisation du projet et la qualité (structurelle, fonctionnelle, esthétique) du produit réalisé. Des rétroactions s'appuyant sur la documentation et sur l'évaluation de prototypes permettent de briser l'apparente rigidité du projet et de faciliter la démarche de création.

Néanmoins, l'application d'une telle méthodologie au sein de groupes de production, qui peut paraître au premier abord aller à contre courant des habitudes non-verbales de création des professionnels, nécessite de reposer sur une véritable volonté collective dans l'organisation. L'enjeu de ces prochaines années dans la profession réside donc dans la formalisation d'un cadre plus général à l'entreprise, capable de susciter l'intérêt et la motivation des acteurs des projets en faveur de ce changement d'état d'esprit.

\section{NOTES}

1. Leleu-Merviel S., La conception en communication. Méthodologie qualité. Hermès, Paris, 1997.

2. Escarpit R., L'information et la communication, théorie générale. Hachette, Paris, 1991.

3. Leleu-Merviel S., La scénistique, méthodologie pour la conception de documents en media multiples suivant une approche Qualité, habilitation à diriger des recherches ; Université de Paris VIII, SaintDenis, 1996.

4. Mucchielli A., Les Sciences de l'Information et de la Communication, Collection Les fondamentaux. Hachette, Paris, 1995.

5. Durand A., Modélisation moléculaire, vers un nouvel outil d'aide à la conception multimedia, Thèse de doctorat en Sciences de l'information et de la Communication, Université de Valenciennes et du Hainaut-Cambrésis, Valenciennes, 1997.

6. Balpe J-P., Hyperdocuments hypertexte hypermedia, Eyrolles, Paris, 1990.

7. Card S., Moran T., Newell A., The psychology of human-computer interaction, Lawrence Erlbaum Associates Publishers, London, 1983.

8. Référence le Larousse 1996.

9. Richard J-F., «Résolution de problèmes: stratégies et impasses », Sciences Humaines, $n^{\circ} 56$, décembre 1995.

10. Huart J., Kolski G, Leleu-Merviel S., «Problèmes de création en multimédia : marier l'expérience de l'audiovisuel et la rigueur de la qualité ", Cahiers du CIRCAV, n 12, à paraître.

11. Durand A., Huart J., Leleu-Merviel S., « Vers un modèle de programme pour la conception de document », in Hypertextes et Hypermedias, Volume $1 \mathrm{~N}^{\circ}$ 1/1997, Hermès, Paris, 1997.

12. Olsina L. A., « Applying the flexible process model to build hypermedia

products », Hypertextes et Hypermedias H²PTM'97, Volume 1 n²-3-4/1997, Hermès, Paris, 1997.

13. AFNOR NF-X-50-120

14. Cartier A., Séminaire Qualité, Publication CNES, Support de cours, Paris, 1992. 


\section{AUTEURS}

JULIEN HUART

Société LOGICA

\section{ALAIN DURAND}

Laboratoire des Sciences de la Communication, Université de Valenciennes et du Hainaut-Cambrésis

SYLVIE LELEU-MERVIEL

Laboratoire des Sciences de la Communication, Université de Valenciennes et du Hainaut-Cambrésis 